\title{
\%
}

Miguel Ángel Martínez Rolland*

\section{DIEZ AÑOS DE RUMANÍA EN LA UNIÓN EUROPEA. BALANCE ECONÓMICO}

Rumanía ha experimentado un fuerte progreso económico desde que se decidió su entrada en la Unión Europea. Su crecimiento ha sido el mayor de los países emergentes de Europa desde la época preadhesión, pero se vio fuertemente afectado por la crisis internacional en el periodo 2009-2012, que estalló poco después de su entrada en la UE. El balance de la entrada en la UE es muy positivo, con un crecimiento económico diferencial, mayor que el de sus vecinos, y un anclaje sólido de las políticas económicas y de las instituciones. Por otro lado, la emigración ha sido particularmente intensa y la demografía constituye un reto para el futuro del país. Los otros grandes retos son la construcción de infraestructuras, muy negativamente afectada por dificultades en los pagos por parte del sector público, y la prestación de servicios públicos en sanidad y educación.

Palabras clave: convergencia, países emergentes, fondos estructurales, infraestructuras, emigración. Clasificación JEL: H54, O52.

\section{Introducción}

Rumanía era uno de los países de Europa del Este para los que la Unión Europea representaba una mayor esperanza tras el «panorama desolador» ${ }^{1}$ en el plano económico que había dejado el régimen comunista, liderado por Nicolae Ceaucescu hasta 1989. La transición a la economía de mercado fue traumática para una industria rumana particularmente obsoleta y vulnerable a la competencia internacional, y las reformas en los años noventa fueron muy lentas y erráticas. Con el comienzo de

\footnotetext{
*Técnico Comercial y Economista del Estado. Consejero Económico y Comercial de España en Rumanía.

Versión de junio de 2017.

1 En palabras de Daianu (1999).
}

las negociaciones para la adhesión a la Unión Europea (UE) y la firma de la asociación con la Unión en el año 2000 se abría un campo de oportunidades para cumplir las esperanzas que no se habían podido realizar.

En este artículo examinaremos hasta qué punto se han aprovechado las oportunidades que ha suministrado la Unión Europea en el plano económico y cuáles son los mayores retos pendientes. En este análisis cabe comparar Rumanía con los países del entorno de Europa del Este y con otros países emergentes, que suministran puntos de referencia útiles. Esta comparación requiere un análisis estructural más detallado de las circunstancias particulares de Rumanía, incluyendo un examen de un fenómeno que ha caracterizado $\triangleright$ 
la evolución reciente del país y que tiene grandes efectos económicos: la emigración. Concluiremos con un examen de los principales retos a los que hace frente Rumanía para la convergencia económica con el resto de la UE, en tres áreas clave como son las infraestructuras, los servicios públicos y la Administración.

\section{Evolución económica de Rumanía desde el periodo preadhesión}

La economía rumana, desde principios de siglo, se ve marcada por la perspectiva de entrada en la Unión Europea, pero también por la coyuntura económica global. En los primeros años del siglo, los países de la antigua Europa del Este gozan de elevados crecimientos económicos y de fuertes entradas de capitales. Pero poco después de la entrada de Rumanía en la Unión, en 2007 , se produce una fuerte crisis económica internacional, que afecta a Europa en dos oleadas, la primera desde 2008-2009 con origen en los Estados Unidos, y la segunda en 2012 con la crisis de la zona euro.

Rumanía exhibió un crecimiento económico extraordinario en la época preadhesión, alcanzando niveles superiores al 8 por 100 interanual, comparables al progreso de los países asiáticos en el mismo período o al de la década dorada del crecimiento de España: los años sesenta.

El crecimiento se interrumpe de forma abrupta con la crisis internacional, que afecta de forma particularmente dura a la Europa Oriental emergente. Las entradas de capitales internacionales en Rumanía en la época preadhesión habían sido masivas, por la perspectiva de un rápido progreso económico y la confianza en el potencial de un país con muchas $\triangleright$

TABLA 1

CRECIMIENTO DEL PIB REAL INTERANUAL DE RUMANÍA

(En porcentaje)

\begin{tabular}{|c|c|c|c|c|c|c|c|c|c|c|c|c|c|c|c|c|c|}
\hline & 2000 & 2001 & 2002 & 2003 & 2004 & 2005 & 2006 & 2007 & 2008 & 2009 & 2010 & 2011 & 2012 & 2013 & 2014 & 2015 & 2016 \\
\hline Crecimiento PIB real (\%) & 2,9 & 5,6 & 5,2 & 5,5 & 8,4 & 4,2 & 8,1 & 6,9 & 8,5 & $-7,1$ & $-0,8$ & 1,1 & 0,6 & 3,5 & 3,1 & 3,9 & 4,8 \\
\hline
\end{tabular}

\section{GRÁFICO 1}

CRECIMIENTO DEL PIB REAL INTERANUAL DE RUMANÍA

(En porcentaje)

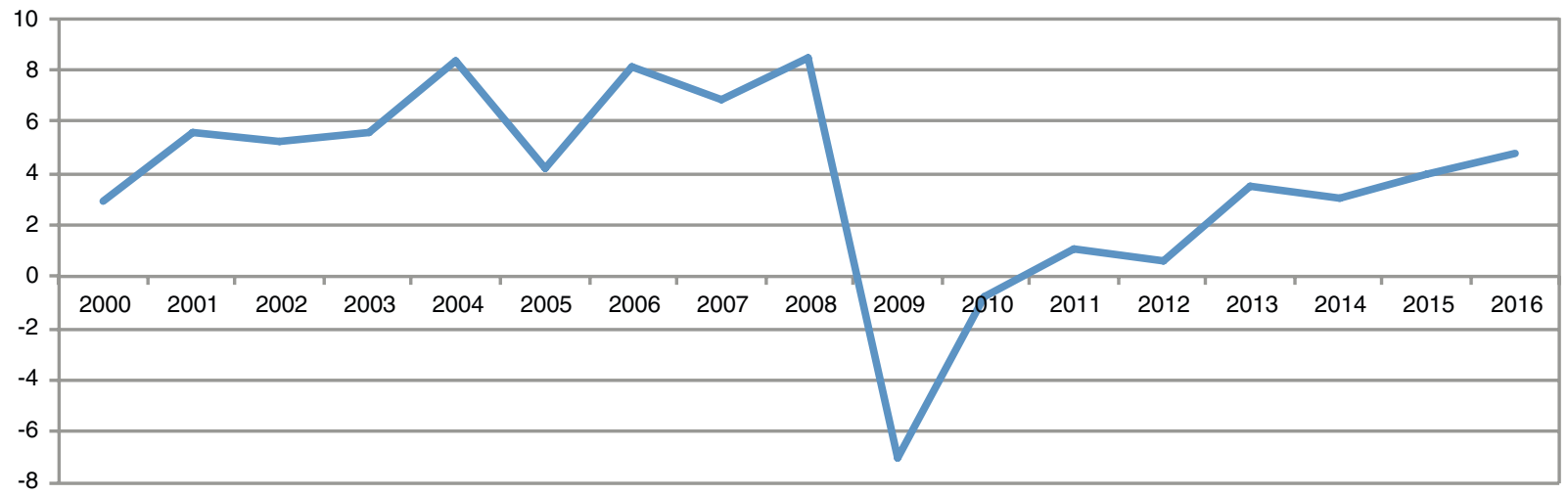

Fuente: FMI, World Economic Outlook Database (abril de 2017). 
TABLA 2

SALDO DE LA CUENTA CORRIENTE DE LA BALANZA DE PAGOS

(En porcentaje del PIB)

\begin{tabular}{|c|c|c|c|c|c|c|c|c|c|c|c|c|c|c|c|c|c|}
\hline & 2000 & 2001 & 2002 & 2003 & 2004 & 2005 & 2006 & 2007 & 2008 & 2009 & 2010 & 2011 & 2012 & 2013 & 2014 & 2015 & 2016 \\
\hline Cuenta corriente (\% PIB) & $-2,6$ & $-3,2$ & $-2,7$ & $-4,5$ & $-7,5$ & $-8,7$ & $-10,4$ & $-13,8$ & $-11,8$ & $-4,8$ & $-5,1$ & $-4,9$ & $-4,8$ & $-1,1$ & $-0,7$ & $-1,2$ & $-2,4$ \\
\hline
\end{tabular}

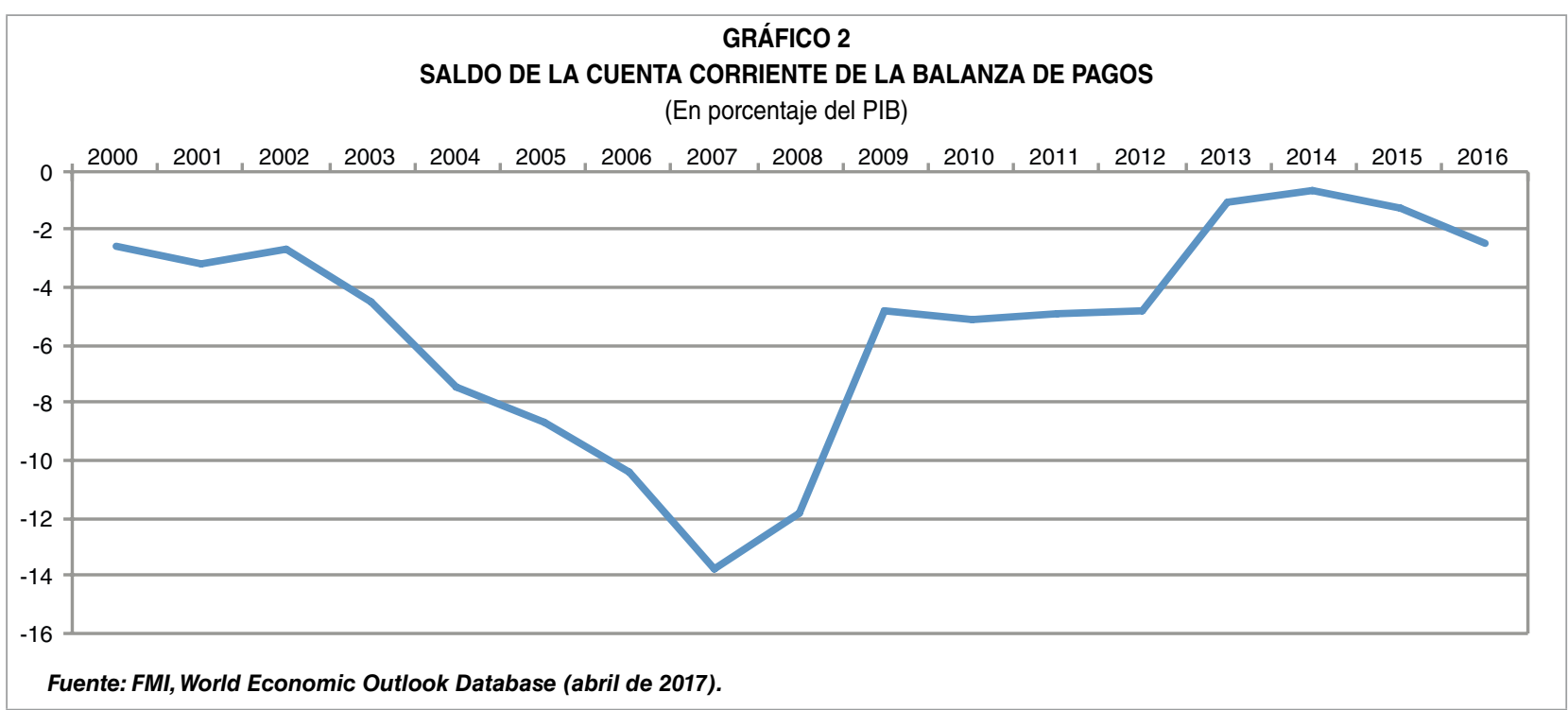

oportunidades por desarrollar. Las entradas de capitales fueron tan intensas que desequilibraron la economía, particularmente al apreciar notablemente el tipo de cambio (Gráfico 3), crear una fuerte burbuja inmobiliaria y un exceso de gasto y de demanda interna que llevó a déficits exteriores descomunales, rozando el 14 por 100 del PIB en 2007 (Tabla y Gráfico 2). \

GRÁFICO 3

TIPO DE CAMBIO: LEU RUMANO / EURO

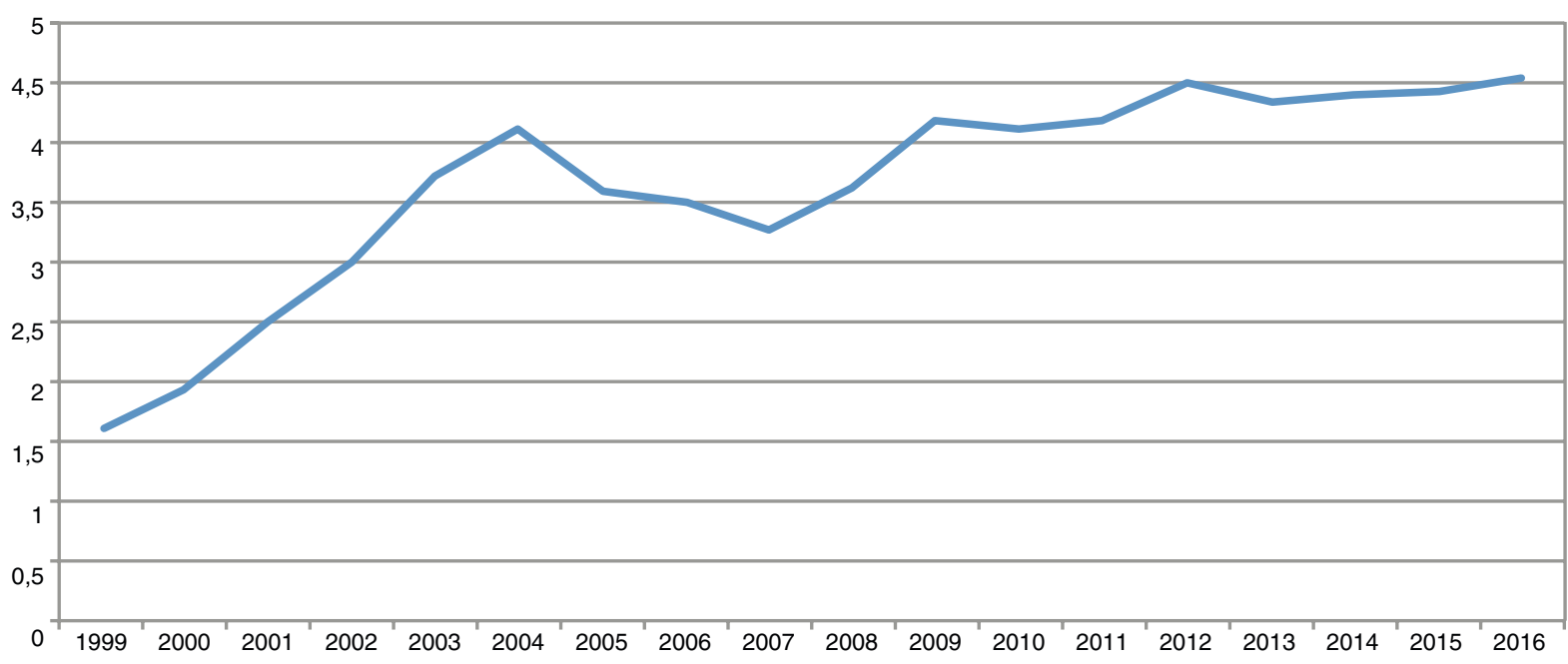

Nota: un aumento significa depreciación del leu rumano y una bajada, una apreciación de la moneda rumana respecto al euro. Fuente: Banco Mundial, Banco Nacional de Rumanía (2017). 
Como consecuencia de la profundidad de estos desequilibrios, la recesión de 2009 en Rumanía fue muy aguda, con una contracción del PIB de más de un 7 por 100. La brusca disminución de la demanda y de la inversión motivó un ajuste exterior muy fuerte. Rumanía tuvo que recurrir a la ayuda exterior y obtuvo un programa de apoyo financiero con el FMI (Acuerdo de Derechos de Giro-Stand By Arrangement) a principios de 2009, acompañado de un paquete de ayuda europea bajo el mecanismo de Apoyo de Balanza de Pagos de la UE².

Un factor que resulta llamativo de la crisis rumana en 2009 es que no vino acompañada de una crisis bancaria, pese a haber sufrido una burbuja inmobiliaria mayor, por ejemplo, que la que experimentó España y un ajuste de precios inmobiliarios más brusco. Podemos mencionar dos razones para este hecho distintivo. En primer lugar, la burbuja inmobiliaria vino financiada por inversión directa del exterior. Muchos inversores extranjeros (incluyendo pequeños y medianos inversores españoles) compraron directamente activos inmobiliarios en Rumanía, esperando una revalorización a raíz de la entrada en la UE. En otros países como Irlanda o España los capitales exteriores no adquirieron directamente los activos, sino que financiaron a los bancos domésticos que efectuaron la expansión de crédito inmobiliario. $Y$ en segundo lugar, la ayuda exterior fue particularmente acertada,

\footnotetext{
2 El paquete total de apoyo ascendió a casi 20.000 millones de euros, aportando el FMI 12.900 millones de euros; la UE, 5.000 millones de euros; el Banco Mundial, 1.000 millones de euros; y la combinación del Banco Europeo de Inversiones (BEI), Banco Europeo de Reconstrucción y Desarrollo (BERD) y Corporación Financiera Internacional, otros 1.000 millones de euros. El programa del FMI entró en vigor en mayo de 2009, cuando recibió un primer desembolso. Es significativo que el FMI tomara un liderazgo tan claro en el rescate, pese a que Rumanía ya formaba parte de la UE. La mayor experiencia del FMl en el país, su prestigio y las limitaciones presupuestarias del mecanismo de ayuda de balanza de pagos de la UE fueron factores determinantes.
}

al promover mediante la llamada «Iniciativa de Viena», de enero de 2009, la coordinación de los principales bancos extranjeros presentes en Europa del Este para que no retiraran fondos de la región y mantuvieran capitalizadas sus filiales y sucursales. En contraste, dentro de la zona euro se produjo, en 2012, un grave fallo de coordinación entre supervisores bancarios de los distintos países, que desencadenó masivas salidas de capitales cuyas consecuencias fueron críticas.

Con todo, la crisis que afectó a Rumanía fue aguda y el crecimiento no se comenzó a recuperar hasta 2013. Los sucesivos Gobiernos se vieron obligados a adoptar durante la crisis rigurosos planes de ajuste macroeconómico, incluyendo reducciones en los salarios públicos de más de un 20 por 100. No fue necesario inyectar capital público en el sector financiero y la deuda pública se mantuvo en niveles moderados - aunque se triplicó desde un 13 por 100 del PIB en 2007 hasta un 39 por 100 en 2013-. El país estuvo bajo programas con el FMI desde 2009 hasta 2015, aunque en la práctica a partir de 2013 no le fueron necesarios.

Es en 2013 cuando el crecimiento retoma con fuerza, por encima de un 3 por 100, y se acelera hasta niveles cercanos al 5 por 100 en 2016 y en el primer trimestre de 2017. Rumanía exhibió el año pasado el mayor crecimiento de la UE. Por un lado, el país se benefició del saneamiento que habían realizado anteriores Gobiernos, pero también a partir de 2015 comienza un tono expansivo de las políticas económicas, que se ha ido intensificando en el último año, hasta el punto que la Comisión Europea ha dictaminado en el informe del Semestre Europeo de 2017 que la economía está sobrecalentada, ligeramente por encima de su potencial. 


\section{Efectos de la entrada en la Unión Europea. Comparativa internacional}

\subsection{Tendencias estructurales}

El análisis de la evolución económica de Rumanía muestra que el periodo preadhesión, con la perspectiva de entrada en la Unión Europea, tuvo un efecto más claro de impulso sobre el crecimiento que el periodo posterior a la adhesión. La crisis internacional dominó la evolución de la economía y, de hecho, el sobrecalentamiento de la inversión provocado por los anormalmente bajos tipos de interés internacionales y por unas perspectivas excesivamente optimistas sobre el efecto de la entrada en la UE motivaron un ajuste particularmente brusco una vez que se produjo el parón en la financiación exterior.

En este contexto, se hace particularmente necesario un análisis cualitativo y comparativo de los efectos de la entrada de Rumanía en la UE sobre algunas de las principales tendencias estructurales de la economía.

En primer lugar, cabe destacar el fuerte incremento del comercio internacional de la economía rumana, que exhibe crecimientos anuales de dos dígitos hasta la crisis internacional, y que se recupera con fuerza después.

Gran parte de este crecimiento consiste en la creación de comercio con la UE, y Rumanía gana cuota de mercado de forma consistente, hasta llegar a duplicar su peso en el comercio intracomunitario en menos de diez años. $\triangleright$

TABLA 3

EVOLUCIÓN DEL COMERCIO INTERNACIONAL DE RUMANÍA

\begin{tabular}{|c|c|c|c|c|c|c|c|c|c|c|c|c|c|c|c|c|c|}
\hline & \multicolumn{17}{|c|}{ Comercio de bienes y servicios (variación interanual \%) } \\
\hline & 2000 & 2001 & 2002 & 2003 & 2004 & 2005 & 2006 & 2007 & 2008 & 2009 & 2010 & 2011 & 2012 & 2013 & 2014 & 2015 & 2016 \\
\hline Importaciones & 27,5 & 18,7 & 12,3 & 16,5 & 22,1 & 16,0 & 22,6 & 28,8 & 0,2 & $-20,7$ & 12,6 & 10,2 & $-1,8$ & 8,8 & 8,7 & 9,2 & 9,3 \\
\hline Exportaciones & 23,2 & 11,9 & 17,1 & 9,3 & 14,1 & 7,6 & 10,4 & 7,8 & $-3,2$ & $-5,3$ & 15,2 & 11,9 & 1,0 & 19,7 & 8,0 & 5,4 & 7,6 \\
\hline
\end{tabular}

Fuente: FMI, World Economic Outlook Database (abril de 2017).

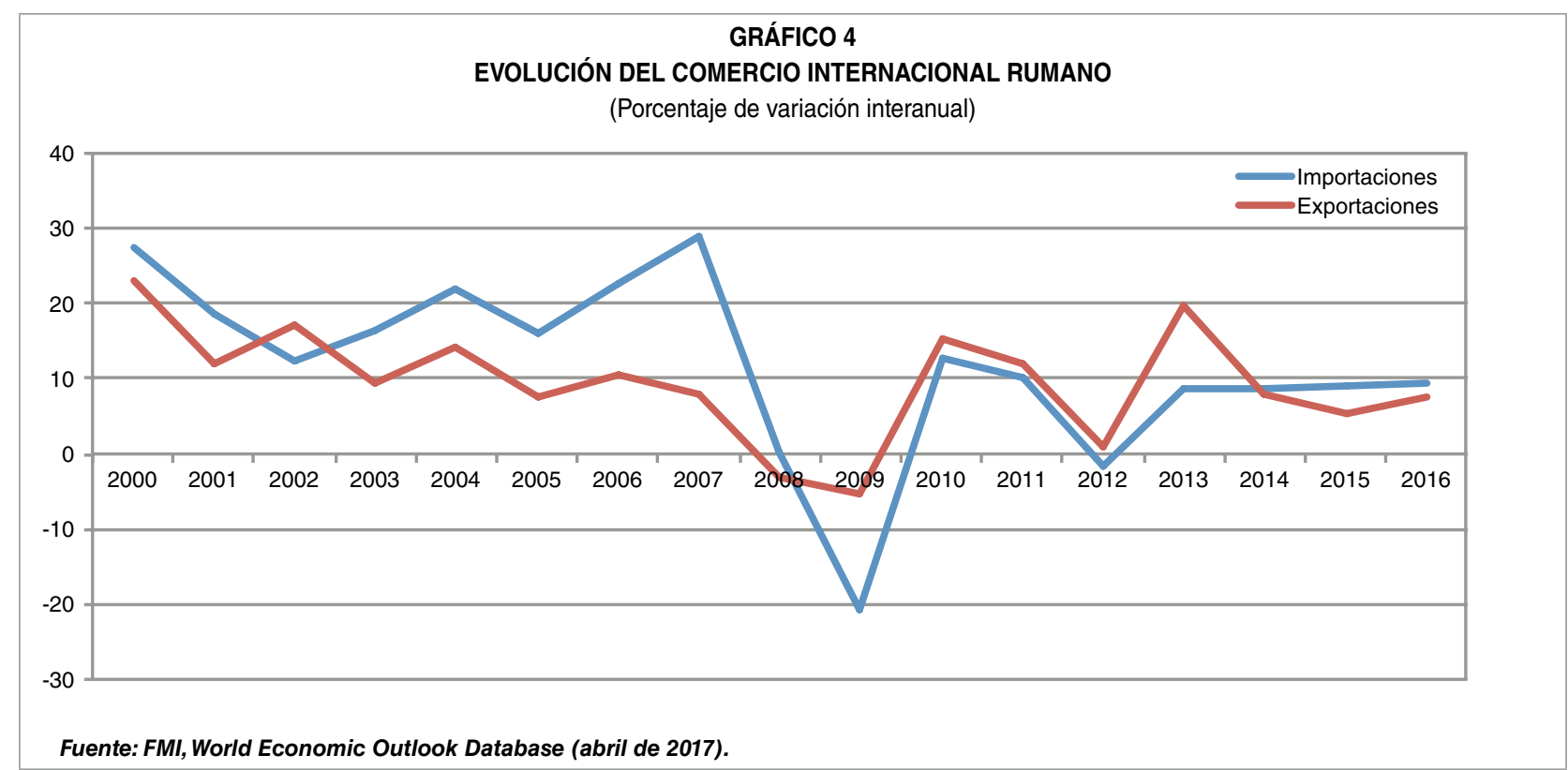




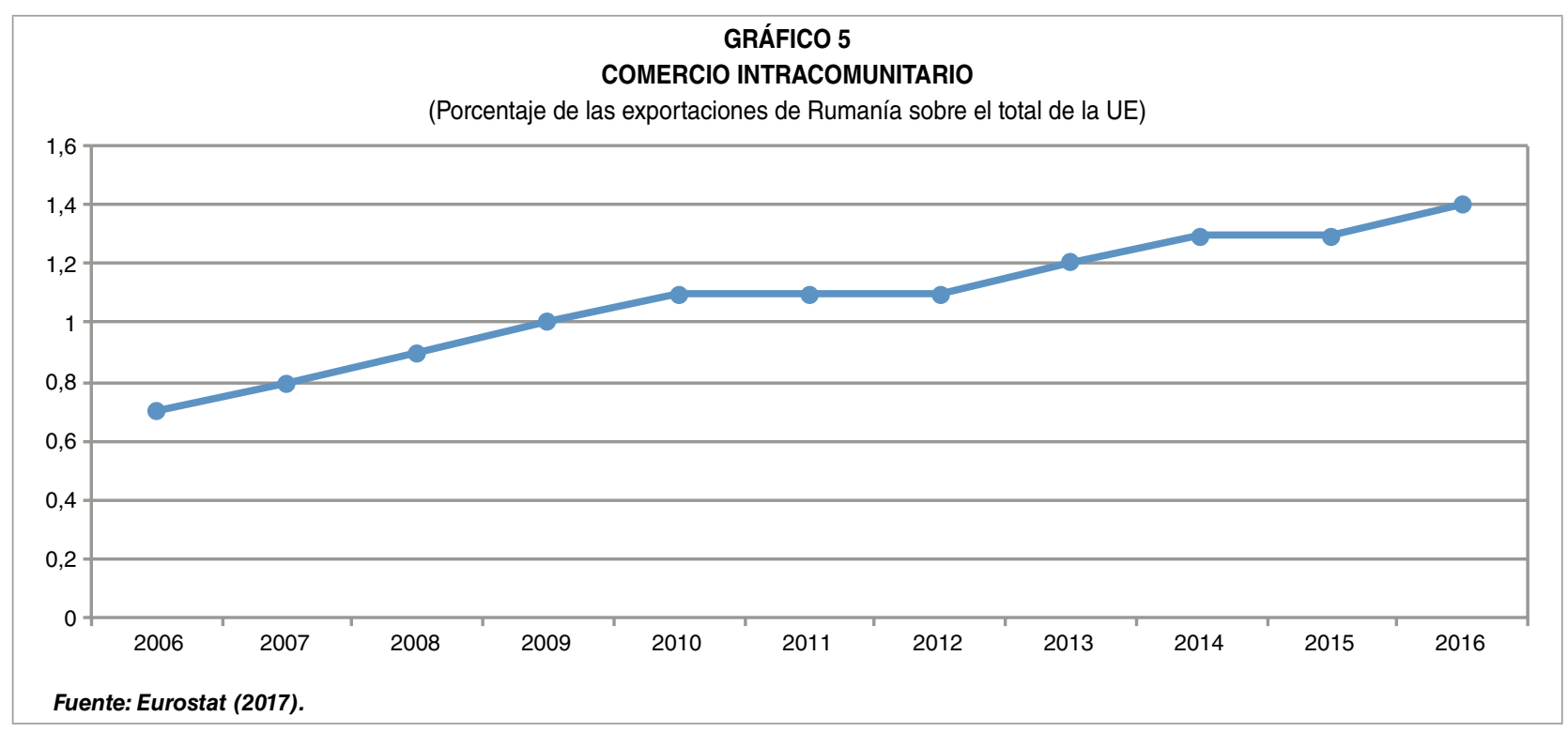

Desde su entrada en la UE, Rumanía ha pasado de constituir el 0,7 por 100 de las exportaciones intracomunitarias al 1,4 por 100 .

La disponibilidad de fondos estructurales (FEDER y FSE) y de fondos de cohesión supone un salto para Rumanía, que pasa a contar en el periodo de las Perspectivas Financieras de la UE 2007-2013 con 20.000 millones de euros, aunque presenta importantes dificultades para gastar adecuadamente esos fondos por problemas internos. Gracias a esos fondos, las infraestructuras progresan, en particular en materia de aguas, aeropuertos y carreteras $^{3}$, aunque de forma más lenta que en otros países. Rumanía partía además de una dotación de infraestructuras muy deficiente.

También la entrada en la UE supuso un anclaje para las políticas económicas, particularmente en materia monetaria y financiera, donde la gestión del Banco Nacional de Rumanía ha sido buena. La inflación se ha reducido de

3 En transportes cabe destacar que mejora la conexión por carreteras con Hungría (en particular para la región de Timisoara) y con Bulgaria (apertura del puente Vidin-Calafat, construido por la empresa española FCC y cuyos accesos fueron levantados por la empresa española AZVI). En materia de aguas se han realizado importantes trabajos en materia de depuración. forma duradera, y el sector financiero no ha requerido una reestructuración a gran escala, aunque ha experimentado unas tasas relativamente elevadas de morosidad a consecuencia de la crisis y de una legislación concursal (quiebras e insolvencias) bastante laxa.

\subsection{Comparativa internacional}

¿Cómo ha sido la evolución de Rumanía en comparación con el resto de la región de Europa Central y Oriental? Muy notable. De hecho, si contamos el periodo preadhesión, Rumanía es el país que más ha crecido en renta per cápita de la región. Aunque partía de niveles de renta muy bajos a principios de siglo, como un país de renta media baja (apenas 1.670 USD per cápita), ha conseguido multiplicar casi por seis el ingreso medio nacional en lo que llevamos de siglo, un progreso extraordinario, comparable incluso al de China y otros países de Asia. Ahora bien, el grueso de la convergencia hacia unos niveles más elevados de renta se produjo antes de la crisis internacional. 
GRÁFICO 6

EVOLUCIÓN DE LA INFLACIÓN EN RUMANÍA, MEDIDA POR LA VARIACIÓN INTERANUAL DEL IPC

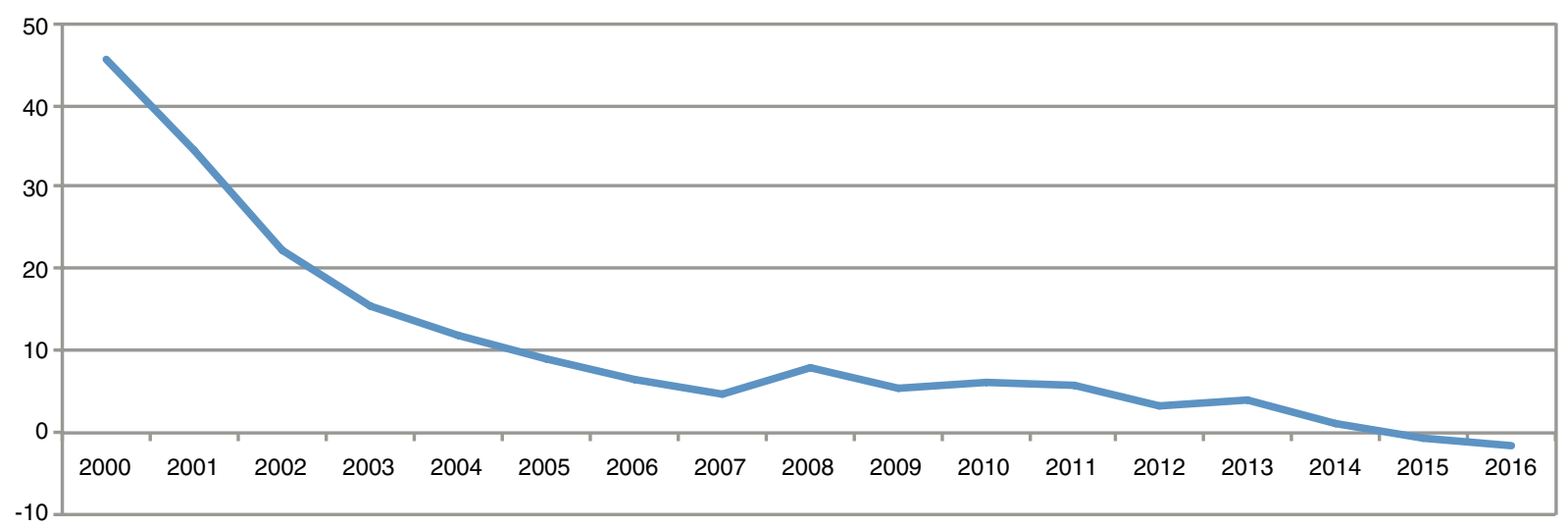

Fuente: FMI, World Economic Outlook Database (abril de 2017).

Rumanía tenía un importante potencial de convergencia (catch-up growth) por partir de niveles muy bajos de renta, pero otros países que partían de niveles similares y que no han entrado en la UE, como Albania o BosniaHerzegovina, no han experimentado el mismo crecimiento. Bulgaria, también nuevo miembro de la Unión, es de los pocos que ha obtenido un crecimiento de una magnitud comparable, aunque menor que Rumanía.

Estos datos parecen apuntar a un efecto económico diferencial de la integración en la UE respecto a otros países de la región. Si además analizamos cualitativamente la evolución de las instituciones de los países (que son fundamentales para el crecimiento a largo plazo), Rumanía ha mostrado una convergencia hacia los niveles de la Unión Europea en los principales ámbitos. En particular, ha mejorado considerablemente en materia de corrupción, como lo reflejan los índices de transparencia internacional ${ }^{4}$, y en la calidad del sistema

4 Rumanía pasó del puesto 63 en el ranking de Transparencia Internacional sobre percepciones de corrupción en el año 1999, con una nota de 3,3, a una nota de 3,7 en 2007 y al puesto 57 en el año 2016 (el sistema de calificación ha cambiado, y la nota del país es de 48). judicial, monitorizado mediante el Mecanismo de Cooperación y Verificación (MCV) de la Unión Europea.

Rumanía ha sabido atraer un tejido importante de inversión extranjera, en particular en el sector de la automoción, con una red de productores de componentes para automóviles muy desarrollada, atraída por las fábricas de Dacia (del grupo Renault) y de Ford. Esta nueva industria de capital extranjero ha podido absorber la reconversión industrial de la obsoleta industria pesada. Otro sector en el que Rumanía ha brillado es en el de la programación y la tecnología de comunicaciones, con un clúster de servicios informáticos en torno a la ciudad de Cluj y sus universidades.

Junto al progreso en renta per cápita, el aspecto más importante de la evolución de Rumanía en el presente siglo es la emigración. Rumanía ha perdido oficialmente a más de un 15 por 100 de su población desde 1990, una tasa muy elevada y preocupante para el país - aunque por otro lado ha podido contribuir a la elevación de la renta per cápita-. Esta pérdida de población ha sido particularmente intensa en los nuevos integrantes de la UE, Rumanía y $\triangleright$ 
TABLA 4

PIB PER CÁPITA EN LOS PAÍSES DE LA EUROPA EMERGENTE

(En USD)

\begin{tabular}{|l|c|c|c|c|c|c|}
\hline \multicolumn{1}{|c|}{ Países } & $\mathbf{2 0 0 0}$ & $\mathbf{2 0 0 7}$ & $\mathbf{2 0 1 3}$ & $\mathbf{2 0 1 6}$ & $\mathbf{2 0 1 6 / 2 0 0 0}$ & $\mathbf{2 0 1 6 / 2 0 0 7}$ \\
\hline Albania & 1.128 & 3.594 & 4.413 & 4.203 & $373 \%$ & $141 \%$ \\
Bosnia-Herzegovina & 1.469 & 3.912 & 4.682 & 4.308 & $293 \%$ & $136 \%$ \\
Bulgaria & 1.614 & 5.813 & 7.703 & 7.369 & $457 \%$ & $166 \%$ \\
Croacia & 4.970 & 13.544 & 13.574 & 12.095 & $243 \%$ & $106 \%$ \\
Hungría & 4.618 & 13.829 & 13.592 & 12.778 & $277 \%$ & $112 \%$ \\
Macedonia & 1.878 & 4.076 & 5.240 & 5.263 & $280 \%$ & $157 \%$ \\
Montenegro & 1.608 & 5.965 & 7.189 & 6.629 & $412 \%$ & $151 \%$ \\
Polonia & 4.476 & 11.257 & 13.777 & 12.316 & $275 \%$ & $137 \%$ \\
Rumanía & $\mathbf{1 . 6 7 0}$ & $\mathbf{8 . 1 2 5}$ & $\mathbf{9 . 5 6 8}$ & $\mathbf{9 . 4 6 5}$ & $\mathbf{5 6 7 \%}$ & $\mathbf{1 6 3 \%}$ \\
Serbia & 1.239 & 5.486 & 6.352 & 5.376 & $434 \%$ & $130 \%$ \\
Turquía & 4.219 & 9.563 & 12.395 & 10.743 & $255 \%$ & $136 \%$ \\
\hline Fuente: FMI, World Econom & & &
\end{tabular}

Bulgaria, cuyos salarios domésticos eran muy bajos. La diferencia de niveles salariales combinada con la progresiva liberalización del movimiento de personas desde principios de siglo han sido los principales factores ${ }^{5}$.

En términos relativos podemos ver (Gráfico 7) que, de los países del este de la UE, Polonia es el único que ha conseguido mantener relativamente estable su población, mientras que, de los países de fuera de la UE, Turquía destaca por experimentar un fuerte crecimiento demográfico desde 1990. Rumanía y Bulgaria muestran una evolución de la población muy similar, con una caída continuada tanto en el periodo preadhesión como posteriormente a su entrada en la UE.

Esta tendencia tan marcada y tan paralela en los países que entraron en la UE en 2007 no parece casual: los datos apuntan a que la entrada en la UE ha tenido un impacto que ha acentuado la emigración ${ }^{6}$.

5 Klaver (2016). The influence of Romanian Migration on the Romanian Economy.

6 Solamente encontramos entre los países de la antigua Unión Soviética una reducción similar de población en Ucrania, que ha perdido casi un 13 por 100 de sus residentes desde 2000 (8 por 100 desde 2007), pero con la excepcionalidad de ser un país que ha experimentado una
La posibilidad de emigrar con menores barreras dentro de la UE ha supuesto un importante incremento de oportunidades para los ciudadanos rumanos que, además, han podido enviar remesas a su país de origen, financiando inversiones, oportunidades educativas y aportando conocimientos adquiridos en el extranjero. Ha supuesto también una válvula de escape para los problemas de desempleo durante la crisis económica. No obstante, la intensidad de la emigración ha provocado una escasez importante de mano de obra en edad activa en Rumanía, así como la pérdida de muchos profesionales de alta calidad.

\section{Retos y perspectivas de futuro}

El análisis muestra que la entrada en la Unión Europea ha sido el principal anclaje para el extraordinario progreso económico $\square$

guerra civil y la pérdida de una provincia (Crimea). En el caso de Moldavia, los datos oficiales muestran una pérdida de población pequeña desde 2000 (-2,3 por 100), pero la emigración real puede ser mucho mayor (el país recibe en torno a un 25 por 100 de su PIB en remesas de emigrantes, mucho más que Rumanía en términos relativos). 


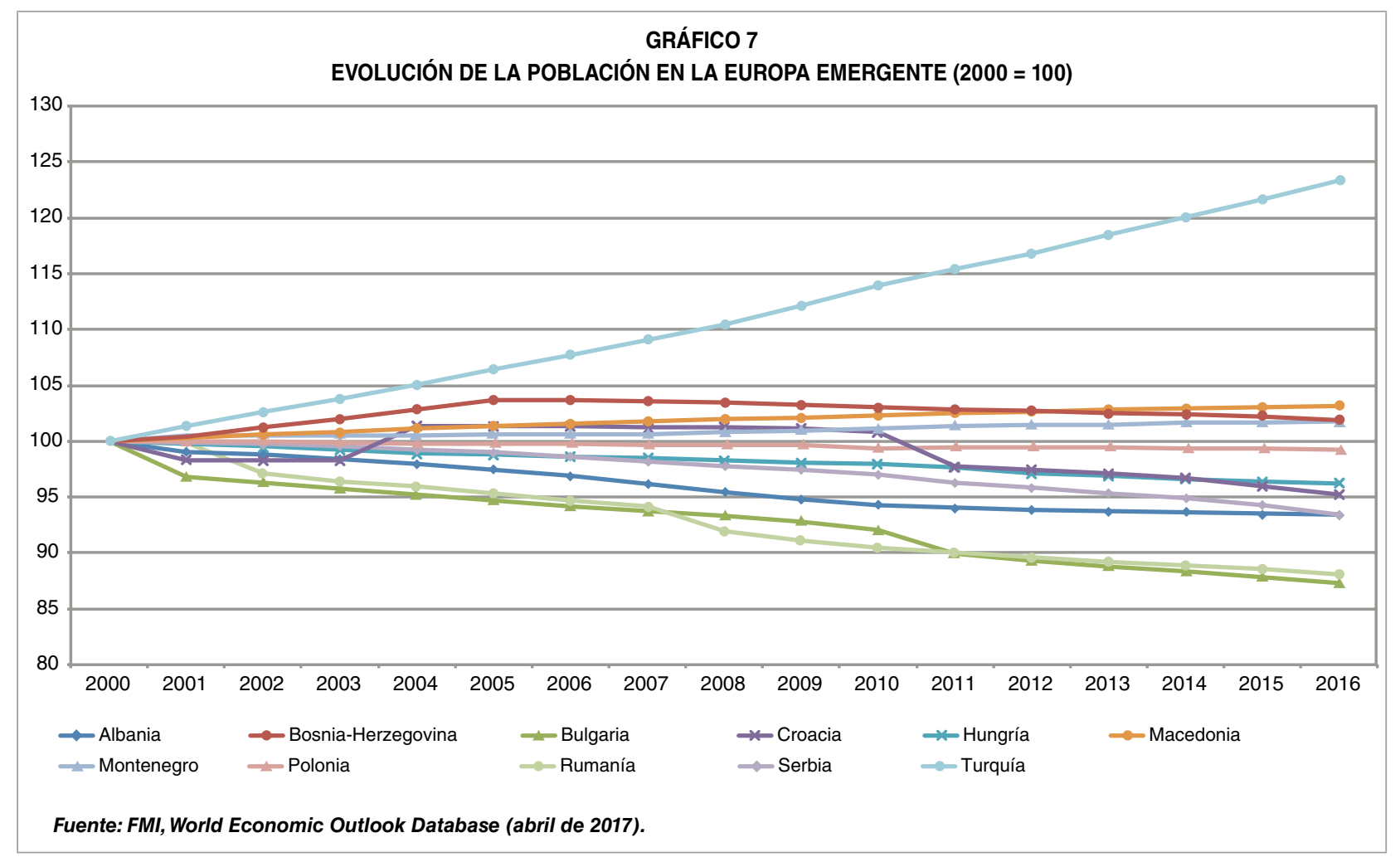

TABLA 5

POBLACIÓN EN LOS PAÍSES DE LA EUROPA EMERGENTE

(En millones)

\begin{tabular}{|c|c|c|c|c|c|c|c|c|}
\hline País & 1985 & 1990 & 2000 & 2007 & 2016 & $\begin{array}{c}\text { Desde } \\
\text { pico }\end{array}$ & $\begin{array}{c}\text { Desde } \\
1990\end{array}$ & $\begin{array}{c}\text { Desde } \\
2007\end{array}$ \\
\hline Albania & 3,059 & 3,306 & 3,089 & 2,970 & 2,885 & $-12,7 \%$ & $-12,7 \%$ & $-2,9 \%$ \\
\hline Bosnia-Herzegovina & - & - & 3,781 & 3,916 & 3,854 & & & $-1,6 \%$ \\
\hline Bulgaria & 8,960 & 8,718 & 8,150 & 7,640 & 7,114 & $-20,6 \%$ & $-18,4 \%$ & $-6,9 \%$ \\
\hline Croacia & - & - & 4,381 & 4,436 & 4,170 & & & $-6,0 \%$ \\
\hline Hungría & 10,599 & 10,375 & 10,222 & 10,066 & 9,835 & $-7,2 \%$ & $-5,2 \%$ & $-2,3 \%$ \\
\hline Macedonia & - & - & 2,010 & 2,045 & 2,073 & & & $1,4 \%$ \\
\hline Montenegro & - & - & 0,612 & 0,616 & 0,623 & & & $1,1 \%$ \\
\hline Polonia & 37,327 & 38,186 & 38,263 & 38,125 & 37,967 & $-1,8 \%$ & $-0,6 \%$ & $-0,4 \%$ \\
\hline Rumanía & 22,971 & 23,459 & 22,435 & 21,131 & 19,760 & $-15,8 \%$ & $-15,8 \%$ & $-6,5 \%$ \\
\hline Serbia & - & - & 7,516 & 7,382 & 7,021 & $-7,2 \%$ & & $-4,9 \%$ \\
\hline Turquía & 49,605 & 52,896 & 64,729 & 70,586 & 79,815 & & $50,9 \%$ & $13,1 \%$ \\
\hline
\end{tabular}

de Rumanía en el siglo xxI. El grueso de la convergencia se produjo en el periodo preadhesión, alimentada por las perspectivas de integración, y en los años inmediatamente posteriores a la entrada en la UE. El crecimiento económico se vio interrumpido por la severa crisis de 2009, pero ha retornado con fuerza desde 2013.

La intensidad de la emigración, combinada con la baja natalidad y su efecto demográfico sobre Rumanía, constituye quizá el mayor reto para el país, que ha perdido una parte $\triangle$ 


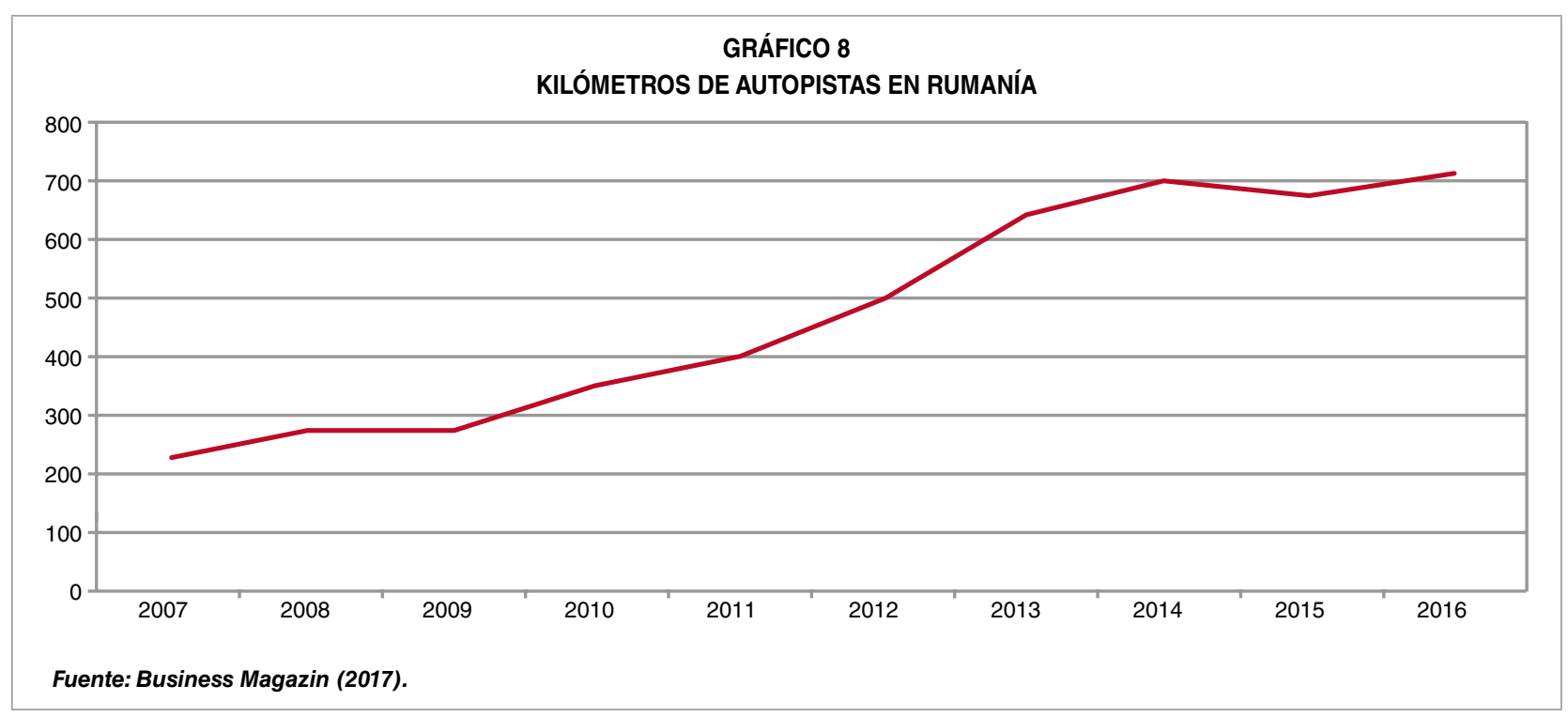

importante de su población en edad activa y en edad fértil. En los poderes públicos comienza a haber conciencia del reto demográfico, pero todavía no constituye una prioridad esencial de las políticas públicas.

A nivel económico estructural, la mayor carencia de Rumanía reside hoy en las infraestructuras. Aunque se han producido progresos en los últimos diez años (como hemos mencionado, en las conexiones con Hungría y Bulgaria, por ejemplo), la mejora va muy despacio. El progreso de las infraestructuras es mucho más lento que el crecimiento económico general.

En particular, la red de autovías y autopistas es muy pequeña, de apenas 700 km (España, con el doble de territorio, contaba con 17.021 en 2015), y la calidad de las carreteras es muy baja. La mortalidad en carretera es la más elevada de la UE junto con la de Bulgaria7 ${ }^{7}$. La red de ferrocarriles también necesita una renovación importante para aumentar su capacidad

\footnotetext{
7 Con 96,8 muertes en carretera anuales por millón de habitantes en 2016, según el European Transport Safety Council, Rumanía sufre la peor siniestralidad de Europa junto a Bulgaria $(99,0)$. Estos niveles triplican a los de España $(38,7)$.
}

y velocidad, actualmente muy lenta. El principal puerto marítimo del país, Constanza, también presenta necesidades de ampliación y modernización.

Pese a la acuciante necesidad de infraestructuras, Rumanía está mostrando serias carencias en la gestión de los importantes fondos europeos que tiene asignados. Una muestra significativa es que, desde 2014, la construcción de kilómetros de autopistas ha sido cercana a cero, pese a la abundancia de fondos europeos.

Los fondos estructurales europeos de la UE disponibles para Rumanía son muy cuantiosos (más de 30.000 millones de euros en el marco financiero 2014-2020), pero al país le está costando asignarlos y gastarlos, en gran parte por las dificultades internas de la Administración rumana.

En materia de inversiones públicas, los problemas de pagos del sector público se han generalizado, debido principalmente al aumento de los retrasos administrativos en los permisos y a la negativa de las Administraciones a reconocer esos retrasos ( $y$ la negativa a reconocer ningún tipo de arbitraje), lo que ha generado $D$ 
un grado de litigiosidad anormalmente elevado $^{8}$. El resultado ha sido la paralización, o un ritmo muy lento, de las obras de infraestructuras, y, en la práctica, la clasificación como contenciosos judiciales de lo que serían atrasos en los pagos a proveedores de infraestructuras. En 2016 y 2017, el gasto en inversión pública se ha contraído notablemente, lo que refleja el agravamiento de la situación.

TABLA 6

PRINCIPALES FONDOS EUROPEOS ASIGNADOS A RUMANÍA EN EL MARCO FINANCIERO 2014-2020

(En millones de euros)

\begin{tabular}{|l|r|}
\hline Fondos Estructurales FEDER & 11.200 \\
Fondo de Cohesión & 8.100 \\
Fondo Agrícola FEADER & 8.127 \\
Fondo Social Europeo FSE & 4.700 \\
Total & $\mathbf{3 2 . 1 2 7}$ \\
\hline Principales programas operativos & \\
Programa Grandes Infraestructuras & 9.419 \\
Desarrollo Rural & 8.128 \\
Desarrollo Regional & 6.700 \\
\hline Fuente: Ministerio Rumano de Fondos Europeos (2017). \\
\hline
\end{tabular}

Parece necesaria una profunda reforma institucional, en particular en el Ministerio de Transportes, y en el marco contractual para las infraestructuras, a fin de alinearlo con estándares internacionales, de modo que Rumanía pueda aprovechar sus fondos europeos y recuperar su atraso relativo en la materia. También parece necesaria una mayor priorización del gasto en infraestructuras en el presupuesto

8 Entre las causas de estos retrasos cabe mencionar la intensidad particularmente elevada de la burocracia (la comisaria rumana de fondos, Corina Cretu, ha dado como ejemplo de burocracia extrema el requisito de presentar 7.000 páginas firmadas a mano para cada proyecto), y el temor de los funcionarios públicos a la rigurosidad de las auditorías del Tribunal de Cuentas y de las investigaciones anticorrupción, que han motivado una actitud muy defensiva a la hora de conceder permisos y asumir responsabilidades. del Estado — cuando la reducción en gasto en inversión pública en estos últimos años ha servido para cuadrar el presupuesto y controlar el déficit-.

A nivel general, la singular complejidad de la burocracia en Rumanía es también un gran reto para el futuro, como menciona la Comisión Europea $^{9}$. La volatilidad normativa, con frecuentes cambios de Gobierno y de ministros, ha contribuido a las dificultades de gestión administrativa.

Rumanía, como hemos mencionado, ha hecho importantes progresos en la lucha contra la corrupción, sobre todo en el sistema judicial, pero continúa siendo un problema económico y social para la población en dos servicios públicos esenciales: sanidad, donde los pagos informales ${ }^{10}$ son todavía frecuentes, y educación.

Rumanía, que es un país muy fértil, tiene potencial para desarrollar más su agricultura -muy condicionada por las carencias del incipiente sistema catastral rumano-y su turismo, actualmente poco explotado. También, en el campo del medio ambiente, existe un importante potencial para avanzar en materia de gestión de residuos, sector en el que la Comisión Europea urge a Rumanía a desarrollar estrategias y proyectos.

A nivel macroeconómico, los dos grandes dilemas son las subidas de salarios y la adopción del euro. Los últimos Gobiernos, al calor del crecimiento económico y de los bajos niveles de desempleo, han adoptado políticas de rentas muy expansivas, con subidas anuales medias de salarios del 13 por 100 en 2016, por encima de la productividad, como $D$

\footnotetext{
9 Recomendación 26 de las CSR (Country Specific Recommendations) de la Comisión Europea para Rumanía.

10 En la práctica, exigencias de pequeños sobornos para la prestación de servicios públicos, una práctica que la Unión Europea considera prioritario extinguir.
} 
advierte el FMI en sus últimas consultas del Artículo IV de 2017. Los fuertes aumentos de salarios públicos y las bajadas de impuestos en los últimos dos años también están presionando las finanzas públicas, y suscitan perspectivas de un ajuste fiscal a medio plazo, a pesar de la baja deuda pública $(39,2$ por 100 del PIB en 2016).

En este contexto, el Banco Central de Rumanía parece querer reservarse la capacidad de ajustar el tipo de cambio en caso de que se confirme una pérdida de competitividad en el medio o largo plazo. Informes como el del miembro del Consejo de Gobierno del Banco Central, Daniel Daianu, apuntan a la necesidad de una convergencia sostenida en la renta de Rumanía antes de contemplar la integración en el área euro. En 2015 la renta media era del 57,5 por 100 de la Unión Europea, y Daianu apunta a la necesidad de consolidar niveles cercanos al 75 por 100 .

\section{Conclusión}

En definitiva, Rumanía ha experimentado un progreso económico extraordinario desde que aseguró su entrada en la Unión Europea a principios de siglo $x \mathrm{xI}$. Este progreso fue particularmente intenso en el periodo preadhesión y en los primeros años de entrada en la UE. Otros países emergentes de Europa del Este han experimentado grandes progresos, pero los de Rumanía han sido particularmente notables. También es necesario recordar que Rumanía partía de niveles bajos de desarrollo económico, y aún sigue siendo el segundo miembro de menor renta per cápita de la Unión Europea.

El apoyo exterior europeo y de las instituciones financieras internacionales, sumado a los esfuerzos de ajuste interno, han permitido que la crisis de 2009 se haya podido superar con una fuerte recuperación del crecimiento. En contraste con la crisis de finales de los noventa, la inflación se ha mantenido bajo control y el sector financiero se ha mantenido estable. Pese a que se ha heredado una economía saneada, mantener la estabilidad macroeconómica ante las presiones fiscales y salariales recientes va a constituir un reto para los próximos Gobiernos.

Rumanía todavía tiene un importante potencial para converger hacia niveles de renta mayores. En particular, existe potencial para una mayor inversión extranjera: Rumanía presenta apenas 3.130 euros de inversión extranjera por trabajador, cuando en Polonia ascienden a 5.053 euros, en Bulgaria son 5.270 euros y en Chequia, 9.703 euros $^{11}$.

No obstante, el crecimiento del país se enfrenta a obstáculos estructurales importantes, como su carencia de infraestructuras y la intensidad de la burocracia. La clave para afrontar los retos futuros puede residir en una reforma administrativa que permita gestionar de forma más eficiente los proyectos de inversión pública, sobre todo las infraestructuras, que cuentan con abundantes fondos europeos, y los servicios públicos en materia de sanidad y educación. Un esfuerzo continuado para reducir la burocracia a muchos niveles también parece necesario. El reto demográfico es quizá el más difícil de afrontar para el país a largo plazo, y debería ser objeto de políticas integrales y de apoyo europeo para afrontar los factores causantes del fenómeno, más allá de las habituales recomendaciones de abordar sus consecuencias mediante recortes de pensiones.

11 Foreign Investors Council (2017). 


\section{Bibliografía}

[1] CASOTA, F. (2017). «2+0+1+7=10 ani de UE» (16-22 de enero). Business Magazin.

[2] COMISIÓN EUROPEA (2017). 2017 European Semester: Country Specific Recommendation I Commission Recommendations - Romania.

[3] DAIANU, D. (1999). Transformarea ca proces real: de la comandã la piatã. Bucarest: IRLI.

[4] DAIANU, D. (coord.) (2016). România și aderarea la zona euro: întrebarea este îN CE CONDIȚII! Institutul European Din Romania.

[5] FONDO MONETARIO INTERNACIONAL (2017). 2017 Article IV Consultation-Staff Report.
[6] FOREIGN INVESTORS COUNCIL (2017). Investitiile Straine Directe: Evolutia si importanta lor in Romania.

[7] KLAVER, F. (2016). The influence of Romanian Migration on the Romanian Economy. Embajada de Holanda en Rumanía.

[8] MURGESCU, B. (2010). «Romania si Europa. Acumularea Decalajelor Economice (15002010)». Polirom.

[9] ROAF, J.; ATOYAN, R.; JOSHI, B. y KROGULSKI, K. (2014). «25 Years of Transition. Post-Communist Europe and the IMF». Regional Economic Issues Special Report. FMI. 


\section{NORMAS DE ESTILO DE PUBLICACIÓN}

La persona o personas interesadas en presentar un artículo para su publicación en el Boletín de Información Comercial Española (BICE) deberán enviar el artículo en formato Microsoft Word a la dirección de correo electrónico revistasice.sscc@comercio.mineco.es

El documento debe cumplir las siguientes características:

1. Ser material original no publicado ni presentado en otro medio de difusión.

2. La extensión total del trabajo (incluyendo cuadros, gráficos, tablas, notas, etcétera) no debe ser inferior a 15 páginas ni superior a 20. La fuente será Times New Roman, tamaño 12 y espaciado doble. Estar paginado en la parte inferior derecha de cada página.

3. En la primera página se hará constar el título del artículo que deberá ser breve, claro, preciso e informativo y la fecha de conclusión del mismo. Nombre y dos apellidos del autor o autores, filiación institucional, dirección, teléfono y correo electrónico de cada uno de ellos, así como la forma en que desean que sus datos aparezcan.

4. En la segunda página del texto se incluirá:

- El título.

- Un resumen del trabajo con una extensión máxima de 10 líneas con la siguiente estructura: objetivo, método y principal resultado o conclusión.

- De 2 a 6 palabras clave que no sean coincidentes con el título.

- De 1 a 5 códigos de materias del Journal of Economic Literature (clasificación JEL) para lo cual pueden acceder a la siguiente dirección electrónica:

https://www.aeaweb.org/jel/guide/jel.php

5. En las siguientes páginas se incluirán el texto, la información gráfica y la bibliografía con la siguiente organización.

- Se incluirá, por este orden, introducción, desarrollo, conclusiones y bibliografía y anexos si los hubiera. Los apartados y subapartados se numerarán en arábigos respondiendo a una sucesión continuada utilizando un punto para separar los niveles de división, según el siguiente modelo:

\section{Título del apartado}

\subsection{Título del apartado}

\subsubsection{Título del apartado}

\section{Título del apartado}

- Las notas de pie de página irán integradas en el texto y su contenido debe estar al final de su misma página en tamaño 10 y espacio sencillo.

- En un archivo Excel independiente se incluirá la representación gráfica (cuadros, gráficos, diagramas, figuras, etc.), que debe llevar título, estar numerada y referenciada en el texto.

En la parte inferior se incluirá la fuente de información y/o notas aclaratorias.

- Las citas de libros y artículos en el texto, se indicarán entre paréntesis con el apellido del autor y el año. Ej.: (Martínez, 1991).

- Las referencias a siglas deben ir acompañadas, en la primera ocasión en que se citen, de su significado completo.

- La bibliografía se ordenará alfabéticamente siguiendo las normas de la American Psychological Association (Harvard-APA): http://cibem.org/paginas/img/apa6.pdf

Libros

APELLIDOS, A.A. (año de publicación). Título del libro (edición) (volumen). Ciudad: Editorial.

Artículo en revista científica

APELLIDOS, A.A. (año de publicación). «Título del artículo». Título de la revista, volumen (número), números de páginas.

\section{Documento en línea}

APELLIDOS, A.A. u ORGANISMO (año, mes de publicación). Título, [en línea]. Ciudad: Editorial. Disponible en:

http://cenamb.rect.ucv.ve/siamaz/dicciona/canaima/canaima2.htm [Recuperado: 2000, 3 de junio]. 